\title{
A comparison of clinical presentation and mri findings in neuromyelitis optica spectrum disorder and spinal cord infarct in complex patients with multiple co-morbidities
}

\begin{abstract}
Neuromyelitis optica spectrum disorder (NMOSD) can be difficult to differentiate from other causes of longitudinally extensive transverse my elites (LETM), such as spinal cord infarct (SCI), especially when patients are complex and have multiple co-morbidities. It is therefore of utility to compare the MRI findings in such cases in order to further distil the key characteristics associated with each condition and improve diagnostic accuracy while predicting variability. This case comparison examines two patients with acute LETM that were followed by a single provider. Each patient's clinical history, cervical and thoracic MRI, CSF studies, AQP4-Ab serology and co morbidities are compared, ultimately leading to the final diagnosis of NMOSD and SCI in each patient respectively. In these patients, clinical history differed in time to nadir and precipitating factor of hypotension. Etiology specific features on MRI were found to be unreliable but associated features helped to synthesize the clinical picture. These difficult cases underscore the need for neurologists to be familiar with the differential diagnosis of LETM and to use key distinguishing findings on MRI to help differentiate NMOSD from other diseases such as SCI.
\end{abstract}

Keywords: longitudinally extensive transverse myelitis, neuromyelitis optica spectrum disorder, spinal cord infarct
Volume 6 Issue 3 - 2017

\author{
Guerrero B,' Amezcua L,' Lerner A² \\ 'Department of Neurology, University of Southern California, \\ USA \\ ${ }^{2}$ Department of Radiology, University of Southern California, \\ USA
}

\section{Correspondence: Guerrero B, Department of Neurology, University of Southern California, USA,}

Email guerrero.brooke@gmail.com

Received: January 27, 2017 | Published: March 03, 2017

\section{Introduction}

When a longitudinally extensive transverse myelitis (LETM) is discovered on MRI in a patient presenting with acute onset motor weakness, a sensory level and loss of sphincter control, NMOSD is usually high on the differential diagnosis since this finding is so frequently encountered in this disease that it has been incorporated into the diagnostic criteria. And yet, distinguishing NMOSD from SCI or the many other causes of acute LETM briefly summarized in (Table 1) is often difficult based on a preceding history that is vague or poorly described and a physical exam confounded by co morbidities (e.g. peripheral neuropathy or peripheral vascular disease). In these cases, it would be beneficial to be able to use etiology specific MRI features, such as the "owl's eye sign" for SCI or "bright spotty lesions" for NMOSD, to differentiate these two diagnoses and promptly pursue appropriate treatment. Many studies, however, have called into question the specificity of such findings, which leaves the neurologist with the task of synthesizing the clinical picture based on multiple modalities. This review will present two cases of LETM and then discuss the key history, physical exam, CSF studies and MRI findings that are typical of NMOSD and SCI. The aim of the comparison is to determine the most reliable clinical findings that help to clarify the diagnosis in the acute setting.

Table I Differential Diagnosis of LETM. ${ }^{3}$

Autoimmune
Neuroinflammatory
Vascular
Infectious
Malignancy
Metabolic

NMOSD, SLE, Antiphospholipid antibody syndrome, Sjogren's syndrome
MS, ADEM, Neuro-Behcet's, Neurosarcoidosis
Spinal dural arteriovenous fistula, Spinal cord infarct
EBV, CMV, HSV,VZV, Syphilis, TB, HIV, HTLV I
Paraneoplastic anti-CRMP-5
BI2 deficiency, Copper deficiency

ADEM:Acute Disseminated Encephalomyelitis;TB:Tuberculosis

\section{Case I}

A 58-year-old African American male with a past medical history of type II diabetes mellitus, benign prostatic hypertrophy, hypertension, and chronic kidney disease (CKD) stage 3 with focal segmental glomerulosclerosis was initially admitted to an outside hospital for anasarca secondary to progression of his CKD from stage 3 to 5 . During his hospital course, he had a decline in mental status and hypotension, which was thought to be secondary to opioids and was treated with naloxone and vasopressors. When the patient awoke, he reported bilateral leg numbness and inability to move his legs or feel his bowel and bladder movements. Outside MRI cervical, thoracic and lumbar spine without contrast revealed longitudinally extensive T2W hyper intense transverse signal with cord expansion and mild degenerative cervical spondylosis. CSF studies were unrevealing and a bone marrow biopsy was negative for malignancy. He received IV Solumedrol $60 \mathrm{mg}$ every 6 hours, later titrated down to prednisone $40 \mathrm{mg}$ PO daily at the time of transfer and also received one day of plasma exchange for presumptive autoimmune LETM. The patient was then transferred to our facility for higher level of care and further work-up of LETM. On admission, he reported sensory loss beginning 
at the nipple line, paralysis of his legs, persistent bowel and bladder incontinence and hand tremor. He also reported severe thoracic back pain that began after his hypotension, worse than his chronic back pain, and recent left knee buckling while walking in the past month. On exam, the patient had intact cranial nerves, a T4 sensory level to all modalities, lower extremity paraplegia with flaccid tone, $3+$ reflexes in the upper extremities, absent reflexes in the lower extremities, silent plantar reflexes, and a fine, postural tremor bilaterally with arms outstretched. Repeat MRI of the brain was unrevealing but MRI of the spinal cord demonstrated persistence of T2W hyper intense transverse signal from T2-T6 with enlargement of the dorsal epidural space and edema of the soft tissues consistent with anasarca (Figure 1A, 1B, \& 2). There was no evidence of abnormal flow voids or disc herniation. CSF studies were also repeated which showed xanthochromia, 2 WBCs, 7 RBCs, elevated glucose $(139 \mathrm{mg} / \mathrm{dL})$, severely elevated protein $(655 \mathrm{mg} / \mathrm{dL})$, oligoclonal bands that were also in the serum, and negative PCR for HSV $1 / 2$ and West Nile virus. Serum ACE and Vitamin B12 were within normal limits and ANA, SSA/B, HTLV I $\mathrm{Ab}$ and $\mathrm{AQP} 4-\mathrm{Ab}$ were negative. The patient was treated with empiric IVIG for 5 days with no improvement. He was referred to physical therapy and at his 2 month follow-up had minimal improvement in his exam.

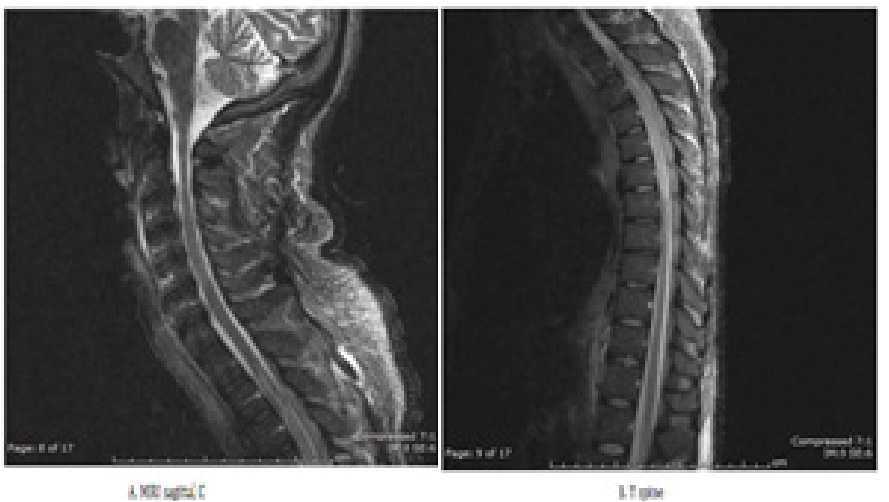

Figure I MRI sagittal $C[A]$ and $T$ spine $[B]$ STIR and T2 weighted images showing central T2 hyperintense lesion of the thoracic cord with associated mild cord expansion extending from T2-T6. There is also enlargement and edema of the dorsal epidural space with responding increased T2 signal in the dorsal epidural fat resulting in narrowing of the thecal sac at T2-T8. Additionally there is edema of the soft tissues consistent with anasarca.

\section{Case 2}

An 81-year-old Caucasian female with a past medical history of systemic lupus erythematosus (SLE), antiphospholipid antibody syndrome, peripheral arterial thrombosis and right occipital stroke presented with ascending numbness and weakness starting in her feet for one week. She stated that it was different from her baseline weakness in the legs and that when the numbness reached her abdomen and she could no longer feel her bowel or bladder movements, she went to the ER. She was initially discharged, but her outside neurologist ordered an MRI of the spinal cord which was suggestive of transverse myelitis. She was then transferred to our facility for higher level of care. Her initial exam revealed profound bilateral hearing loss, left homonymous hemianopsia, a T5 sensory level to all modalities, full upper extremity strength, $4 / 5$ strength in the proximal lower extremity muscle groups, $1 / 5$ strength in the left dorsiflexor, 3+ reflexes throughout and silent plantar reflexes. Repeat MRI of the brain showed encephalomalacia from a chronic right posterior cerebral artery infarct (Figure 3) and MRI of the spinal cord revealed $\mathrm{T} 2 \mathrm{~W}$ hyperintense central cord signal from $\mathrm{C} 7$ to $\mathrm{T} 5$, originally interpreted as having an "owl's eye configuration" and interval decrease in cord swelling and contrast enhancement compared to the prior outside image (Figure 4A \& 4B). She also had severe cervical canal stenosis but the impression by Neuroradiology and Neurosurgery was that her hyperintense signal began beneath this (Figure 5). CSF studies showed clear, colorless CSF with 2 WBCs and no RBCs, normal glucose $(88 \mathrm{mg} / \mathrm{dL})$, normal protein $(51 \mathrm{mg} /$ $\mathrm{dL}$ ) and one oligoclonal band. She had a reactive serum HTLV $1 \mathrm{Ab}$ and a 1:160 titter for HHV-6 IgG but negative CSF PCR for both viruses. She was positive for AQP4-Ab but her ANA titter was $<1: 80$ and lupus anticoagulant was also negative. Vitamin B12 and ACE were within normal limits. The patient was treated with 5 days of IV Solumedrol and discharged to an acute rehabilitation centre before her AQP4-Ab resulted and she did not follow-up. She was re-admitted 3 months later for worsening symptoms. She had remained ambulatory for about a month and was participating in physical therapy but progressively worsened and continued to need a Foley catheter. At the time of re-presentation, her legs were flaccid, she had a complete T5 sensory level and no control over bowel and bladder function. At the outside hospital the patient had received IV Solumedrol prior to transfer. Repeat MRI cervical and thoracic spine showed worsening $\mathrm{T} 2$ hyper intensity from the cervical region to T7.

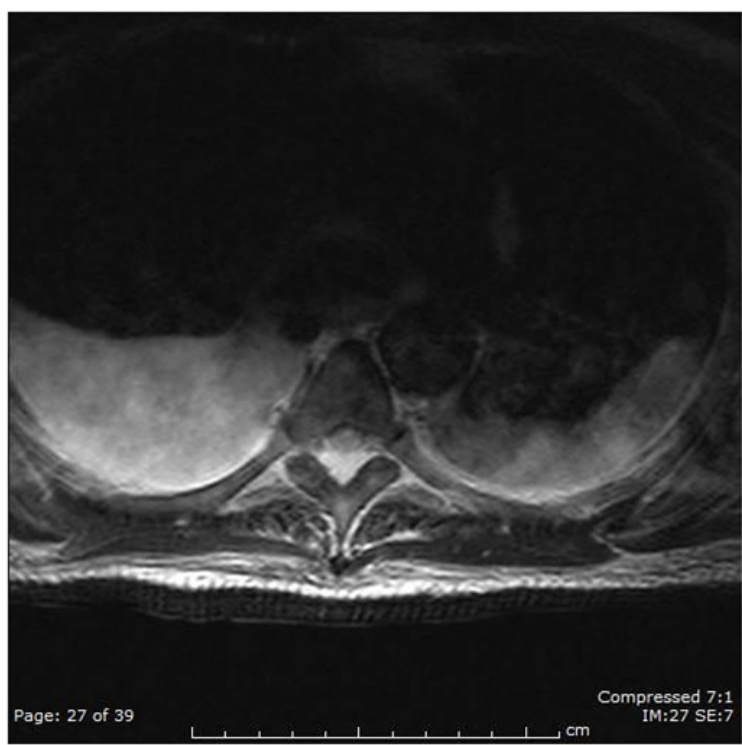

Figure 2 MRI axial T spine T2 weighted image demonstrating ill defined transverse cord hyper intensity and edema of the dorsal epidural space with narrowing of the thecal sac.

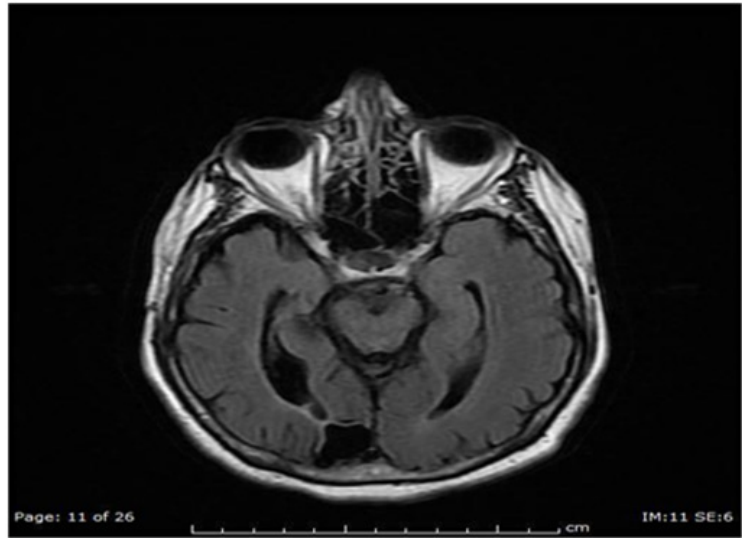

Figure 3 Axial T2 FLAIR image demonstrating posterior cerebral artery territory encephalomalacia from prior stroke. 


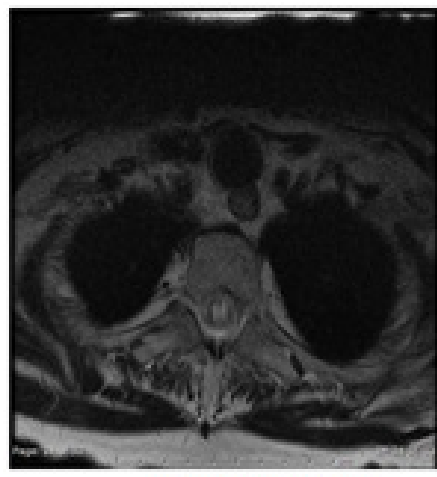

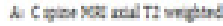

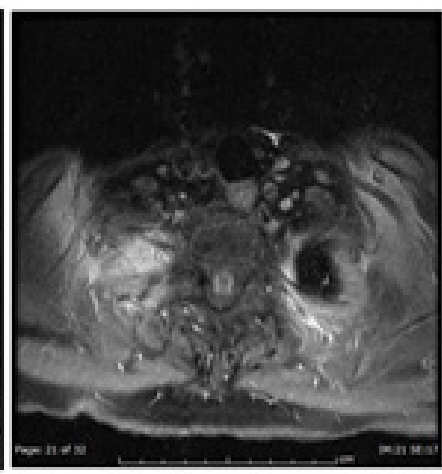

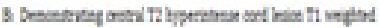

Figure $4 \mathrm{C}$ spine $\mathrm{MRI}$ axial T2 weighted [A] demonstrating centra T2 hyperintense cord lesion TI weighted [B] axial postcontrast image demonstrates peripheral enhancement within the lesion with central area of non-enhancement and TI hypointensity corresponding to T2 hyperintensity seen on $\mathrm{T} 2$ weighted image.

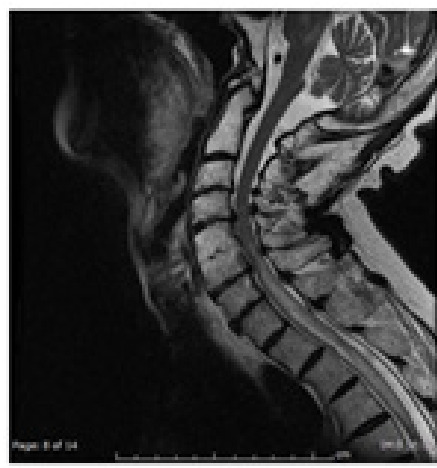

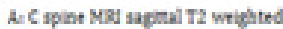

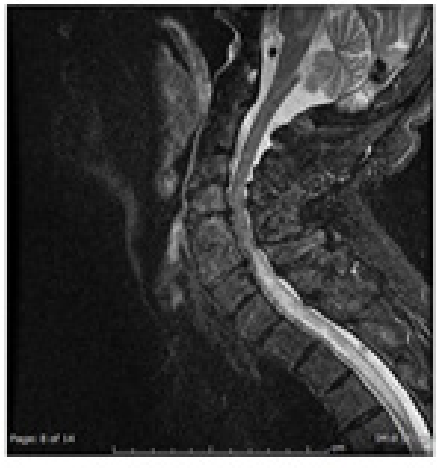

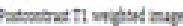

Figure $5 \mathrm{C}$ spine MRI sagittal T2 weighted [A] and STIR [B] images demonstrating extensive longitudinal T2 hyperintense central cord lesion extending from C7 toT5 level and mildly expanding the cord. There is severe multilevel degenerative disc disease at C3-C4 through $\mathrm{C} 6-\mathrm{C} 7$ with degenerative intervertebral fusion of $\mathrm{C} 5-\mathrm{C} 6$ and severe spinal canal stenosis at C6-C7.The cord lesion is thought to be unrelated to the spinal stenosis as it extends far caudad to the level of stenosis. Postcontrast TI weighted [C] image demonstrates heterogeneous enhancement within the lesion.

\section{Discussion}

Neuromyelitis Optica Spectrum Disorder, NMOSD is an inflammatory disorder of the CNS that is believed to be related to the presence of pathogenic aquaporin-4 antibodies (AQP4-Ab). In the spinal cord, AQP4 is localized in perivascular astrocyte foot processes and in the glial limiting membrane. ${ }^{1}$ Studies have also shown that myelin oligodendrocyte glycoprotein (MOG) may also be a pathogenic antigen in AQP4-Ab negative patients. ${ }^{1,2}$ One of the hallmark clinical syndromes suggestive of this disease is LETM, defined as a complete paralysis and sensory loss below the level of the lesion and loss of sphincter control. Pain, pruritus, and paroxysmal tonic spasms are also frequent symptoms experienced by patients. ${ }^{3}$ On MRI, the lesion is typically longitudinally extensive (greater than three vertebral body segments in length) with lesions extending into the medulla having a high predictive value. ${ }^{3}$ Other syndromes include optic neuritis, area postrema syndrome, acute brainstem syndrome, acute diencephalic syndrome, and symptomatic cerebral syndrome. ${ }^{3}$ In order to formally diagnose NMOSD in a patient that is AQP4-Ab positive, only one core clinical characteristic is required. If a patient is AQP4-Ab negative, two core clinical characteristics are required to meet the diagnostic criteria for clinically definite $\mathrm{NMO}$, and one must be eitheroptic neuritis, LETM, or area postrema syndrome. ${ }^{3}$

Similar to most autoimmune disorders, NMOSD has a female predominance with rates reported at $6: 1$ in retrospective reviews. ${ }^{2}$ The average age at onset is older than for MS, ( 40 years), and the race distribution is mixed, although small reviews have reported a higher rates in African Americans and Asians. ${ }^{2,4}$ There is also a confirmed between NMOSD and non-organ specific autoimmune disorders such as SLE, antiphospholipid syndrome, and Sjogren's syndrome, with the presence of AQP4-Ab being specific for the NMOSD phenotype, since it is absent in patients that do not have the neurological symptoms of LETM or optic neuritis. ${ }^{5,6}$ A large series of myelitis in SLE patients found patients to have a monophonic grey matter syndrome with early bladder involvement and rapid are flexic flaccid paraparesis, and a relapsing white matter syndrome with a more mild spastic paraparesis and better recovery. ${ }^{6}$ Of interest, the white matter myelitis syndrome seemed to be more associated with AQP4-Ab seropositivity $(57.1 \%$ versus $12.5 \%$ ) even though central grey matter involvement in NMOSD is common. ${ }^{6}$ LETM in SLE patients therefore seems to be complex product of either the SLE itself or concurrent NMO. The presence of AQP4-Ab has been correlated with both annual relapse rate and higher EDSS scores. ${ }^{7}$ CSF studies in NMOSD patients typically show pleocytosis (neutrophils or eosinophils), elevated protein (sometimes $>1 \mathrm{~g} / \mathrm{L}$ ), and absent oligoclonal bands. ${ }^{7,8}$

\section{Spinal cord infarct}

SCI represents $1 \%$ of all strokes and 5-8\% of acute myelopathies. ${ }^{9}$ Causes are heterogeneous and include aortic surgery, aortic dissection, aortic atherosclerosis, severe hypotension, spinal trauma, surfer's my elopathy, and either cardiogenic or fibro cartilaginous embolism. ${ }^{9}, 10$ Knowledge of spinal vascular anatomy is necessary to identify spinal vascular syndromes. The anterior spinal artery (ASA) supplies the anterior two-thirds of the spinal cord and originates from the vertebral arteries with collateral supply from ridiculer arteries that correspond to functional regions of the spinal cord ${ }^{11}$ (Table 1). Paired posterior spinal arteries (PSAs) originate from either the vertebral arteries or the posterior inferior cerebellar arteries and supply the dorsal one-third of the spinal cord. The area of the cord most susceptible to ischemic injury in the setting of hypotension is T1-T4 due to the large distance between ridiculer arteries. ${ }^{12}$ The classic symptoms of SCI when the anterior spinal artery is involved are sudden ridiculer pain at the level involved, motor weakness, loss of sphincter control, spinothalamic deficits with sparing of dorsal column related vibration and Proprioception. ${ }^{12}$ A retrospective review of 27 patients with confirmed spinal cord ischemia revealed that the majority of patients had anterior spinal artery syndrome with only a minority having transverse infarcts that caused a more severe central cord syndrome. ${ }^{13}$ Symptom onset was usually acute (minutes to hours) with some reporting TIA symptoms in the preceding years. No identifiable cause 
was found in 20 of the patients, but three had an episode of arterial hypotension preceding infarction and three had disk herniation. ${ }^{13}$ CSF was shown to have increased protein but without pleocytosis or oligoclonal bands ${ }^{13}$ (Table 2).

\section{Differentiating NMOSD from SCI based on MRI}

LETM is visualized on MRI as extensive T2W hyperintense signal, typically involving the central cord in NMOSD and matching an arterial territory, usually the ASA, in SCI. ${ }^{4,8}$ The predilection for central cord in NMOSD is likely due to distribution of AQP4, while the variable distribution of SCI (e.g ASA, PSA, central or transverse infarcts) are determined by the artery occluded. Central and transverse infarcts are of particular interest due to their similar appearance to central NMOSD lesions and are thought to occur in the watershed border zone between the territories of the perforating sulcal and circumferential arteries. ${ }^{6}$ The "owl's eye sign" hyper intensity of the ventral horns that occurs with ASA infarct is characteristic, as are the "bright spotty lesions" of NMOSD, defined as a hyperintense spotty lesions on a T2W image almost of the same intensity as CSF but not qualifying as a syrinx, that seem to represent complement mediated damage but are still not fully characterized. ${ }^{8,9,14}$ Concurrent vertebral body infarction is thought to also be a confirmatory finding of SCI but in practice is rare and although DWI can be used, this modality is not as sensitive for SCI as it is for cerebral infarcts. ${ }^{14,4}$ With respect to location along the spinal cord, SCI tends to occur where collateral circulation is poor (midthoracic cord and conus) whereas NMOSD occurs around junctions, specifically cervicomedullary or cardiothoracic, which are areas with abundant collateral circulation. ${ }^{15,16}$ SCI rarely extends to the pial surface and does not usually enhance in the acute phase in contrast to NMOSD which typically enhances due to breakdown of the bloodcord barrier. ${ }^{15}$ A recent study aiming to differentiate NMOSD from other causes identified brainstem extension, cord expansion, centrally located T2 axial lesions that were $>50 \%$ of cord area, "bright spotty lesions" and T1 dark lesions as more commonly associated with NMOSD compared to other etiologies of LETM, such as MS, SCI and spinal dural arteriovenous fistula. ${ }^{7}$ Another study evaluating the MRI characteristics of SCI found that the majority of their patients had infarcts involving the thoracic cord with a "pencil-like" hyperintense lesion in $98.2 \%$ of patients, "owl's eye sign" in only four patients and T1W cord enlargement in $40 \%$ of patients ${ }^{10}$ (Table $3 \& 4$ ).

Table 2 Radicular artery supply to the spinal cord

\begin{tabular}{lll}
\hline Functional Region of Spinal Cord & Radicular Artery Level & Artery of Origin \\
\hline CI-T3 & C3 & Vertebral arteries \\
T3-T7 & C6 or C7 & Cervical ascending arteries \\
T8-cone & T7 *variable & Intercostal artery \\
Cone & T9-L2 (Adamkiewicz artery) & Intercostal or lumbar artery \\
\hline
\end{tabular}

Table 3 Typical distinguishing features of NMOSD and SCI

\begin{tabular}{|c|c|c|c|c|c|}
\hline & MRI Findings & $\begin{array}{l}\text { Typical Spinal } \\
\text { Cord Location }\end{array}$ & $\begin{array}{l}\text { Time to } \\
\text { Nadir }\end{array}$ & Distinct Clinical Features & Demographics \\
\hline NMOSD & $\begin{array}{l}\text { - T2hyperintensity } \\
\text { extends to pial } \\
\text { surface } \\
\text { - Contrast } \\
\text { enhancement } \\
\text { - Centrally located } \\
\text { lesion } \\
\text { - Lesion >50\% of } \\
\text { cord area } \\
\text { - TI dark spots } \\
\text { - "Bright spotty } \\
\text { lesion" }\end{array}$ & $\begin{array}{l}\text { Cervico- thoracic } \\
\text { or cervico- } \\
\text { medullary } \\
\text { junction }\end{array}$ & 8 days & $\begin{array}{l}\text { - Paroxysmal tonic spasms } \\
\text { - Area postrema syndrome }\end{array}$ & $\begin{array}{l}\text { Non-Caucasian } \\
\text { females }\end{array}$ \\
\hline $\mathrm{SCl}$ & $\begin{array}{l}\text { - Pencil-like T2 } \\
\text { hyperintensity with } \\
\text { "owl's eye sign" on } \\
\text { axial cut and cord } \\
\text { swelling (ventral } \\
\text { horns) } \\
\text { - Increased vertebral } \\
\text { bone marrow } \\
\text { signal } \\
\text { - Persistent root/ } \\
\text { ventral cord } \\
\text { enhancement } \\
\text { several months } \\
\text { after event }\end{array}$ & Lower thoracic & $<4$ hours & $\begin{array}{l}\text { - Sudden flaccid paralysis } \\
\text { - Areflexia } \\
\text { - Loss of sensation to pain/temperature } \\
\text { with preserved vibration and } \\
\text { Proprioception } \\
\text { - Autonomic deficits } \\
\text { - Severe radicular pain }\end{array}$ & Caucasian males \\
\hline
\end{tabular}


Table 4 Case comparison between two clinical cases of LETM

\begin{tabular}{|c|c|c|c|c|c|c|c|}
\hline & Demographics & AQP4Ab & OCBs & Time to nadir & MRI & Co-morbidities & Initial Exam \\
\hline $\begin{array}{l}\text { Case } \\
\text { I }\end{array}$ & $\begin{array}{l}58 \text { y/o African American } \\
M\end{array}$ & & - & $<24 \mathrm{~h}$ & $\begin{array}{l}\text { Transverse T2 } \\
\text { hyperintensity T2-T8 } \\
\text { Edema of dorsal } \\
\text { epidural space }\end{array}$ & $\begin{array}{l}\text { ESRD } \\
\text { BPH } \\
\text { HTN } \\
\text { DM II }\end{array}$ & $\begin{array}{l}\text { Motor: } \\
\text { UE 5/5 } \\
\text { LE 0/5 all groups } \\
\text { Sensory:T4 } \\
\text { LE DTRs absent } \\
\text { Plantar reflex silent }\end{array}$ \\
\hline $\begin{array}{l}\text { Case } \\
2\end{array}$ & 82 y/o Caucasian F & + & + & $\sim$ I month & $\begin{array}{l}\text { Central cord T2 } \\
\text { hyperintensity C7-T5 } \\
\text { "Owl's eye sign" } \\
\text { Contrast enhancement }\end{array}$ & $\begin{array}{l}\text { HTLV + } \\
\text { Cervical stenosis } \\
\text { Anti- } \\
\text { phospholipid } \\
\text { syndrome } \\
\text { SLE } \\
\text { Stroke } \\
\text { PAD }\end{array}$ & $\begin{array}{l}\text { Motor: } \\
\text { UE } 5 / 5 \\
\text { LE } 4 / 5 \text { except I/5 in } \\
\text { left DF } \\
\text { Sensory:T5 } \\
\text { LE DTRs 3+ } \\
\text { Plantar reflex silent }\end{array}$ \\
\hline
\end{tabular}

\section{Conclusion}

These two cases are representative of how difficult the initial clinical picture can be in patients with multiple co-morbidities and incidental confounding findings during the work-up. Both patients had multiple vascular risk factors and an acute onset of symptoms, but the SCI patient's time to nadir was shorter than the NMOSD patient (within 24 hours versus 1 month). The SCI patient had a preceding episode of hypotension combined with systemic vascular disease and anasarca while the NMOSD patient had a more insidious onset of symptoms but also had a history of antiphospholipid antibody syndrome associated with prior stroke and peripheral arterial thrombosis, making the possibility of SCI likely in both cases. Neither patient had CSF pleocytosis but there were oligoclonal bands in the NMOSD patient, which is not normally characteristic, and a markedly elevated protein level in the SCI patient, which has been documented.

The MRI comparison was limited by the inability to give the the SCI patient contrast due to his co-morbid ESRD, but the NMOSD patient's lesion did appear to involve the central cord of the cervicothoracic junction with contrast enhancement while the SCI patient's lesion was in the thoracic region most susceptible to hypotensive injury and had more of an ill defined transverse appearance. It is interesting to note that the NMOSD patient actually had the "owl's eye sign" that is normally associated with SCI and also had incidental cervical canal stenosis that was not felt to be the cause of her cord signal. She was also initially positive for HTLV I Ab and her ANA and lupus anticoagulant were negative, calling into question her prior diagnoses and making her case very difficult to interpret initially. These cases demonstrate that both time to nadir and subtle differences in the distribution of a LETM can be used to differentiate SCI from NMOSD. However, the caveat remains that with the current limitations of neuroimaging and the presence of overlapping risk factors, these diseases can still present in very similar ways and appear the same on MRI

\section{Acknowledgments}

None.

\section{Conflicts of interest}

None.

\section{Funding}

None.

\section{References}

1. Papadopoulos MC, Verkman AS. Aquaporin water channels in the nervous system. Nat Rev Neurosci. 2013;14(4):265-277.

2. Sato DK, Callegaro D, Lana-Peixoto MA, et al. Distinction between MOG antibody-positive and AQP4 antibody-positive NMO spectrum disorders. Neurology. 2014;82(6):474-481.

3. Mealy MA, Wingerchuk DM, Greenberg BM, et al. Epidemiology of neuromyelitis optica in the United States: a multicenter analysis. Arch Neurol. 2012;69(9):1176-1180.

4. Sand IK. Neuromyelitis Optica Spectrum Disorders. Continuum (Minneap Minn). 2016;22(3):864-896.

5. Pekcevik Y, Mitchell CH, Mealy MA, et al. Differentiating neuromyelitis optica from other causes of longitudinally extensive transverse myelitis on spinal magnetic resonance imaging. Mult Scler. 2016;22(3):302-311.

6. Kitley JL, Leite MI, George JS, et al. The differential diagnosis of longitudinally extensive transverse myelitis. Mult Scler. 2012;18(3):271-285.

7. Nardone R, Fitzgerald RT, Bailey A, et al. Longitudinally extensive transverse myelitis in systemic lupus erythematosus: case report and review of the literature. Clin Neurol Neurosurg. 2015;129:57-61.

8. Yonezu T, Ito S, Mori M, et al. "Bright spotty lesions" on spinal magnetic resonance imaging differentiate neuromyelitis optica from multiple sclerosis. Mult Scler. 2014;20(3):331-337.

9. de Sèze J, Kremer L, Collongues N. Neuromyelitis optica spectrum disorder(NMOSD): A new concept. Rev Neurol (Paris). 2016;172(4-5):256-262.

10. Rabinstein AA. Vascular myelopathies. Continuum (Minneap Minn). $2015 ; 21: 67-83$.

11. Weidauer S, Nichtweiß M, Hattingen E, et al. Spinal cord ischemia: aetiology, clinical syndromes and imaging features. Neuroradiology. 2015;57(3):241-257.

12. Novy J, Carruzzo A, Maeder P, et al. Spinal cord ischemia: clinical and imaging patterns, pathogenesis, and outcomes in 27 patients. Arch Neurol. 2006;63(8):1113-1120.

13. Vongveeranonchai N, Zawahreh M, Strbian D, et al. Evaluation of a patient with spinal cord infarction after a hypotensive episode. Stroke. 2014;45(10):e203-205.

14. Romi F, Naess H. Spinal Cord Infarction in Clinical Neurology: A Review of Characteristics and Long-Term Prognosis in Comparison to Cerebral Infarction. Eur Neurol. 2016;76(3-4):95-98. 
15. Hyun JW, Kim SH, Jeong IH, et al. Bright spotty lesions on the spinal cord: an additional MRI indicator of neuromyelitis optica spectrum disorder? J Neurol Neurosurg Psychiatry. 2015; 86(11):1280-1282.

16. Kister I, Johnson E, Raz E, et al. Specific MRI findings help distinguish acute transverse myelitis of Neuromyelitis Optica from spinal cord infarction. Mult Scler Relat Disord. 2016;9:62-67.
17. Chang KH, Lyu RK, Chen CM, et al. Distinct features between longitudinally extensive transverse myelitis presenting with and without anti-aquaporin 4 antibodies. Mult Scler. 2013;19(3):299-307.

18. Faig J, Busse O, Salbeck R. Vertebral body infarction as a confirmatory sign of spinal cord ischemic stroke: report of three cases and review of the literature. Stroke. 1998;29(1):239-243. 